\title{
Article
}

\section{Intramolecular Janus Segregation of a Heteroarm Star Copolymer}

Yung Chang, Wen-Chang Chen, Yu-Jane Sheng, Shaoyi Jiang, and Heng-Kwong Tsao

Macromolecules, 2005, 38 (14), 6201-6209 • DOI: 10.1021/ma050596g

Downloaded from http://pubs.acs.org on November 24, 2008

\section{More About This Article}

Additional resources and features associated with this article are available within the HTML version:

- $\quad$ Supporting Information

- $\quad$ Links to the 5 articles that cite this article, as of the time of this article download

- $\quad$ Access to high resolution figures

- $\quad$ Links to articles and content related to this article

- $\quad$ Copyright permission to reproduce figures and/or text from this article

\section{View the Full Text HTML}




\title{
Intramolecular Janus Segregation of a Heteroarm Star Copolymer
}

\author{
Yung Chang, ${ }^{\dagger, \ddagger}$ Wen-Chang Chen, ${ }^{\dagger}$ Yu-Jane Sheng, ${ }^{\dagger}$ Shaoyi Jiang, ${ }^{\ddagger}$ and \\ Heng-Kwong Tsao*,
}

Department of Chemical Engineering, National Taiwan University, Taipei, Taiwan 106, R.O.C; Department of Chemical Engineering, University of Washington, Seattle, Washington 98195; and

Department of Chemical and Materials Engineering, National Central University, Jhongli,

Taiwan 320, R.O.C

Received March 21, 2005; Revised Manuscript Received May 13, 2005

\begin{abstract}
Conformations of heteroarm star copolymers $A_{n} B_{n}$ are investigated by off-lattice Monte Carlo simulations. Intramolecular segregation is always observed in selective solvents. It can also be found in a common good solvent, which however gives block incompatibility. The degree of Janus segregation is characterized by the mean-square separation between $A$ and $B$ blocks, $\left\langle\left(\mathbf{x}_{A}-\mathbf{x}_{B}\right)^{2}\right\rangle$, or by the orientation correlation between the two blocks, $\left\langle\mathbf{x}_{A} \cdot \mathbf{x}_{B}\right\rangle$, where $\mathbf{x}_{i}$ denotes the relative position of the center of mass associated with the block arms $i$ from the star core. The degree of segregation grows with increasing arm numbers while the effect of arm lengths is insignificant. The influence of the intramolecular segregation on the aggregation behavior in solution can be revealed through interactions between two star copolymers. The potential of mean force and second virial coefficient are calculated for various solvent qualities. In a selective solvent, the blockarm star copolymer can form a unimolecular micelle structure and always shows intermolecular repulsions. In contrast, the heteroarm star copolymer may display the Janus segregation, which leads to intermolecular attractions. This consequence facilitates the formation of multimolecular micelles.
\end{abstract}

\section{Introduction}

Star-shape block copolymers possess the ability to display different morphologies in polymer solutions, in bulk state as well as at the interface due to their complex architecture. This unique property allows them to become potential materials for many applications such as nanocarriers for drug delivery ${ }^{1-3}$ and fabrication of smart polymer films. ${ }^{4}$ Various molecular architectures of star copolymers have been reported ${ }^{5-9}$ including heteroarm $A_{n} B_{n}$, blockarm $(A B)_{n}$, and miktoarm $A_{n} B_{m}$ structures. For heteroarm and miktoarm diblock star polymers, two different kinds of arms emanate from the same core. Each arm is composed of only $A$ - or $B$-type monomers. A heteroarm star copolymer bears an equal number of $A$ and $B$ arms such as polystyrene/poly(2-vinylpyridine) $\left(\mathrm{PS}_{7}-\mathrm{PVP}_{7}\right)$ while a miktoarm star has asymmetric $A$ and $B$ arms. On the other hand, a blockarm $(A B)_{n}$ star copolymer is composed of $n$ diblock arms with one of the blocks as the inner section of the arms.

In a selective solvent, linear block copolymers may self-assemble to form multimolecular micelles, which exhibit a core-shell structure. The worse solubilized blocks constitute the core while the better solubilized blocks form the shell. Recently, it is reported that heteroarm star copolymers are also associated to form micelles. However, the critical micelle concentration (cmc) of the star copolymer is much higher than that observed for the linear copolymer. For example, the cmc of $\mathrm{PS}_{6}-\mathrm{PVP}_{6}$ in toluene is $7.4 \times 10^{-4} \mathrm{~g} / \mathrm{cm}^{3}$, while that of the linear copolymer having the same block length is $2.2 \times 10^{-7} \mathrm{~g} / \mathrm{cm}^{3}, 3$ orders of magnitude lower..$^{10}$ This consequence indicates that the difference in architecture

\footnotetext{
$\dagger$ National Taiwan University.

$\$$ University of Washington.

$\S$ National Central University.

* Corresponding author. E-mail: hktsao@cc.ncu.edu.tw.
}

between star and linear copolymers results naturally in diverse unimolecular conformations and therefore their solution behavior.

Single molecular conformations associated with a star copolymer play an important role in determining the aggregation behavior in different solvent qualities. Most of the investigations on morphology of structures formed by macromolecules in solutions were inferred from scattering techniques, viscometry, or cryo-transmission electron microscopy. However, details of morphology cannot be easily extracted for molecules with complex structures. Recently, single molecular morphologies of heteroarm star copolymers were studied by atomic force microscopy (AFM) with molecular resolution. ${ }^{11,12}$ Assuming that the adsorbed molecules still retain their conformations formed in solutions, $\mathrm{PS}_{7}-\mathrm{PVP}_{7}$ was deposited onto mica or Si wafers from different solvent conditions. The experimental results revealed that even the solvent $(S)$ is good for both $A$ and $B$ blocks of a star copolymer; differences in affinity between $A-S$ and $B-S$ induce an intramolecular segregation, leading to the formation of Janus-like conformation. On the other hand, a near-uniform spherical structure is present as the attraction between $A-S$ and $B-S$ becomes comparable. Those works provide us with insightful information on possible conformations associated with heteroarm star copolymers in solutions. Nonetheless, these findings were based on speculation from the observed "hatlike" and "spherical" conformations of the adsorbed star copolymers. It is still doubtful whether or not the polymer conformation in solution is preserved after deposition.

An alternative for studying macromolecular features that are not fully explained by experiments is computer simulation. Monte Carlo (MC) simulations have been performed for homoarm star polymers and provided deeper insight into developing the scaling relations of conformational properties. ${ }^{13-15}$ The conformation prop- 
erties of miktoarm star copolymers in dilute solutions were investigated, and the simulation results were used to examine the validity of certain theoretical models. ${ }^{16}$ Recently, conformations of heteroarm star copolymer in good and selective solvents were studied by lattice MC simulations. In strongly selective solvents, the possibility of the formation of nonspherical structures was suggested. ${ }^{17}$ In this work, we investigate intramolecular segregation phenomena of heteroarm star copolymer due to incompatibility of arms with different block types as well as selective solvents. The effects of arm numbers and lengths on the degree of Janus segregation are studied. To understand the influence of morphology on the solution behavior, the potential of mean force and second virial coefficient are also calculated for various solvent qualities.

\section{Methodology}

The star polymer contains total beads $N=f L+1$, where $f$ represents the arm number and $L$ is the arm length in terms of the number of beads. The interactions between bonded beads are represented by an infinite deep square-well potential $u_{\mathrm{b}}$ as $^{18-20}$

$$
\beta u_{\mathrm{b}}= \begin{cases}\infty & r \geq \varsigma \sigma \\ 0 & \varsigma \sigma>r \geq \sigma \\ \infty & r<\sigma\end{cases}
$$

where $\sigma$ is the bead diameter, $\varsigma=1.4$, and $\beta=1 / k_{\mathrm{B}} T$. $k_{\mathrm{B}}$ denotes the Boltzmann constant and $T$ the temperature. This interaction potential indicates that each randomly selected bead on the star polymer was allowed to move around its position with a restriction of the bond fluctuation between $\sigma$ and $1.4 \sigma$. Nonbonded beads interact through the square-well potential $u_{p q}$,

$$
\beta u_{p q}(r)= \begin{cases}0 & r \geq \lambda \sigma \\ \beta \epsilon_{p q} & \lambda \sigma>r \geq \sigma \\ \infty & r<\sigma\end{cases}
$$

where $p, q=A$ or $B$ and $\lambda=1.5$. Note that the starcore bead is neither $A$ nor $B$ type. It interacts as a hard sphere with all the other beads on the star copolymer. Although no solvent particles are explicitly taken into account in the simulations, this model potential depicts the solvent-mediated short-range interactions. ${ }^{21,22}$ The energy parameter $\epsilon_{p q}\left(\epsilon_{A A}, \epsilon_{B B}, \epsilon_{A B}\right)$, which should be considered as potential of mean force rather than potential energy, is varied to promote repulsion (attraction) in addition to the excluded-volume interaction. For example, in water and other polar solvents, the hydrophilic (polar) segments prefer being surrounded by solvent molecules so that the solvent-mediated interactions between these two segments are effectively repulsive $\left(\epsilon_{p q}>0\right)$. On the contrary, the hydrophobic segments in the polar solvent therefore tend to attract each other $\left(\epsilon_{p q}<0\right)$.

The trial move employed for chains of the equilibration and production process is bead displacement motion. It involves randomly picking a bead and displacing it to a new position in the vicinity of the old position. The distance away from the original position is chosen with a probability that the condition of equal sampling of all points in the spherical shell surrounding the initial position must be satisfied. The new configuration resulting from this move is accepted according to the standard Metropolis acceptance criterion. ${ }^{23}$
The effective interaction between two star copolymers can be manifested through the second virial coefficient $B_{2}$. The virial expansion for the compressibility factor takes the form

$$
\beta P / \rho=1+B_{2} \rho+B_{3} \rho^{2}+\ldots
$$

where $P$ denotes the osmotic pressure, $\rho$ is the molecular number density of the system, and $B_{i}$ represents the $n$th virial coefficients. We adopted the algorithm proposed by Harismiadis and Szleifer ${ }^{24}$ to evaluate $B_{2}$. The second virial coefficient can be calculated in terms of the interaction potentials from standard statistical mechanics $^{25,26}$

$$
B_{2}=2 \pi \int\left(1-\exp \left[-\frac{F_{2}(\xi)-2 F_{1}}{k T}\right]\right) \xi^{2} \mathrm{~d} \xi
$$

where $F_{1}$ is the Helmholtz free energy of a single polymer at infinite dilution in a solvent and $F_{2}(\xi)$ is the Helmholtz free energy of a system composed of the same solvent and two polymers when their center-of-mass distance is $\xi$. The difference in the exponential can be thought of as an effective potential between the polymers, i.e.

$$
U_{\text {eff }}(\xi)=F_{2}(\xi)-2 F_{1}=-k T \ln \left\langle\exp \left[-\beta U_{2}(\xi)\right]\right\rangle
$$

where $U_{2}$ is the intermolecular interaction energy between the two polymers. $\langle\cdots\rangle$ denotes a canonical average over all the configurations of the two polymers. Note that each polymer does not know the presence of the other polymer. A trial move leading to overlap of two star polymers is allowed but yields an infinite value of intermolecular interaction, $U_{2} \rightarrow \infty$.

The algorithm used to calculate $U_{\text {eff }}$ and then $B_{2}$ is as follows: $2 \times 10^{5}$ completely independent polymer conformations are generated by using the Metropolis recipe. ${ }^{23} \mathrm{~A}$ pair of polymers is selected out of $10^{5}$ pairs. One of the pair is placed with its center of mass fixed at $(0,0,0)$. Then place another polymer with its center of mass set at $(\xi, 0,0)$; i.e., two polymers are separated by a distance of $\xi$. The separation $\xi$ is systematically increased during the simulation process to estimate the effective potential, $U_{\text {eff }}(\xi)$. At each separation, 50 random rotations of the second polymer are performed. A total of $5 \times 10^{6} U_{2}$ are sampled at each separation. After the effective potential between the polymers is calculated by eq 4 as a function of $\xi$, the second virial coefficient can be obtained through eq 3 .

\section{Results and Discussion}

The intramolecular segregation may be induced by two mechanisms: the selective solvent and the incompatibility between $A$ and $B$ block arms. These two mechanisms tend to separate arms of $A$ block from arms of $B$ block. On the contrary, the conformation entropy favors complete mixing between those two blocks. The competition between internal energy reduction and entropy effect determines the degree of segregation. Two limits are therefore anticipated: the complete mixing corresponding to the homoarm star polymer and intramolecular Janus segregation corresponding to extreme incompatibility.

The simplest case associated with the selective solvent condition corresponds to $\epsilon_{B B}<0$ and $\epsilon_{A A}=\epsilon_{A B}=0$. The solvent is athermally good for $A$ arms but is relatively 
poor for $B$ arms. In fact, under this condition, the interaction between $A$ and $B$ arms is not simply excludedvolume repulsion but implicitly repulsive. ${ }^{27}$ On the other hand, the simplest case for the block incompatibility is represented by $\epsilon_{A B}>0$ and $\epsilon_{A A}=\epsilon_{B B}=0$. In an athermally good solvent for both $A$ and $B$ arms, there exist solvent-mediated repulsions between $A$ and $B$ arms. For the above-mentioned two mechanisms, the solvent quality for the whole star copolymer is assumed to be away from the poor solvent regime.

Similar to the excluded-volume parameter $1 / 2-\chi$, with $\chi$ denoting the Flory-Huggins interaction parameter, an integral measure of solvent quality ${ }^{21}$ can be defined as

$$
v=\frac{1}{4}\left(b_{A A}+b_{B B}+2 b_{A B}\right)
$$

where $b_{i j}$, representing the partial bare second virial coefficient between segment $i$ and $j$, is normalized as

$$
b_{i j}=\frac{2 \pi \int_{0}^{\infty}\left[1-\exp \left(-\beta u_{i j}\right)\right] r^{2} \mathrm{~d} r}{b_{0}}
$$

Here $b_{0}=2 \pi \sigma^{3} / 3$ represents the second virial coefficient associated with hard spheres. As mentioned earlier, the polymer segments interact through a square-well potential as in eq 2 ; thus

$$
b_{i j}=1+\left(\lambda^{3}-1\right)\left[1-\exp \left(-\beta \epsilon_{i j}\right)\right]
$$

where $\lambda$ and $\epsilon_{i j}$ are the width and the depth of the potential well. For an athermally good solvent $\left(\epsilon_{i j}=0\right)$, one has $b_{i j}=1$ and $v=1$. A phantom chain has $b_{i j}=0$ and $v=0$. If a $\Theta$-solvent displays behavior similar to a phantom chain, a poor solvent corresponds to negative values of $v$. In the present study, the solvent quality associated with block incompatibility case $\left(\beta \epsilon_{A B}>0\right)$ is always athermally good while that of selective solvent case declines with decreasing $\epsilon_{B B}$. Nevertheless, the integral measure of solvent quality $v>0$ as long as $\beta \epsilon_{\mathrm{BB}} \gtrsim-1$.

A. Block Incompatibility. The morphology of a heteroarm star copolymer depends significantly on the solvent-mediated interaction between $A$ and $B$ blocks. The incompatibility between $A$ and $B$ block arms can be realized in terms of repulsion between $A$ and $B$ blocks. Despite of the fact that both blocks are in an athermally good solvent condition $\left(\epsilon_{A A}=\epsilon_{B B}=0\right)$, the solvent-mediated interaction between $A$ and $B$ block arms $\epsilon_{A B}$ is able to induce chain collapse $\left(\epsilon_{A B}<0\right)$ or Janus segregation $\left(\epsilon_{A B}>0\right)$ within a heteroarm star copolymer. When $\beta \epsilon_{A A}=0, \beta \epsilon_{B B}=0$, and $\beta \epsilon_{A B}=-0.611$, one has $b_{A A}=1, b_{B B}=1$, and $b_{A B}=-1$ and thus $v=0$ (eq 5). It implies that for the case of specific interactions between $A$ and $B$ blocks $\beta \epsilon_{A B} \simeq-0.611$ corresponds to a $\Theta$-solvent condition at which the excluded-volume repulsion cancels out the $A-B$ segment attraction. In other words, a solvent which is athermal for $A$ as well as for $B$ obviously is acting like a $\Theta$-solvent for $A B$ block copolymers in (the hypothetical) case of a strongly attractive interaction between $A$ and $B, \beta \epsilon_{\mathrm{AB}} \simeq-0.6$. Note that for a diblock star copolymer there is no exact definition of the theta condition. The condition $v=0$ is merely an indication of the onset of polymer collapsing process. Many parameter combinations can result in theta states (in which $v=0$ ). For example, $\beta \epsilon_{A A}=\beta \epsilon_{B B}$ a
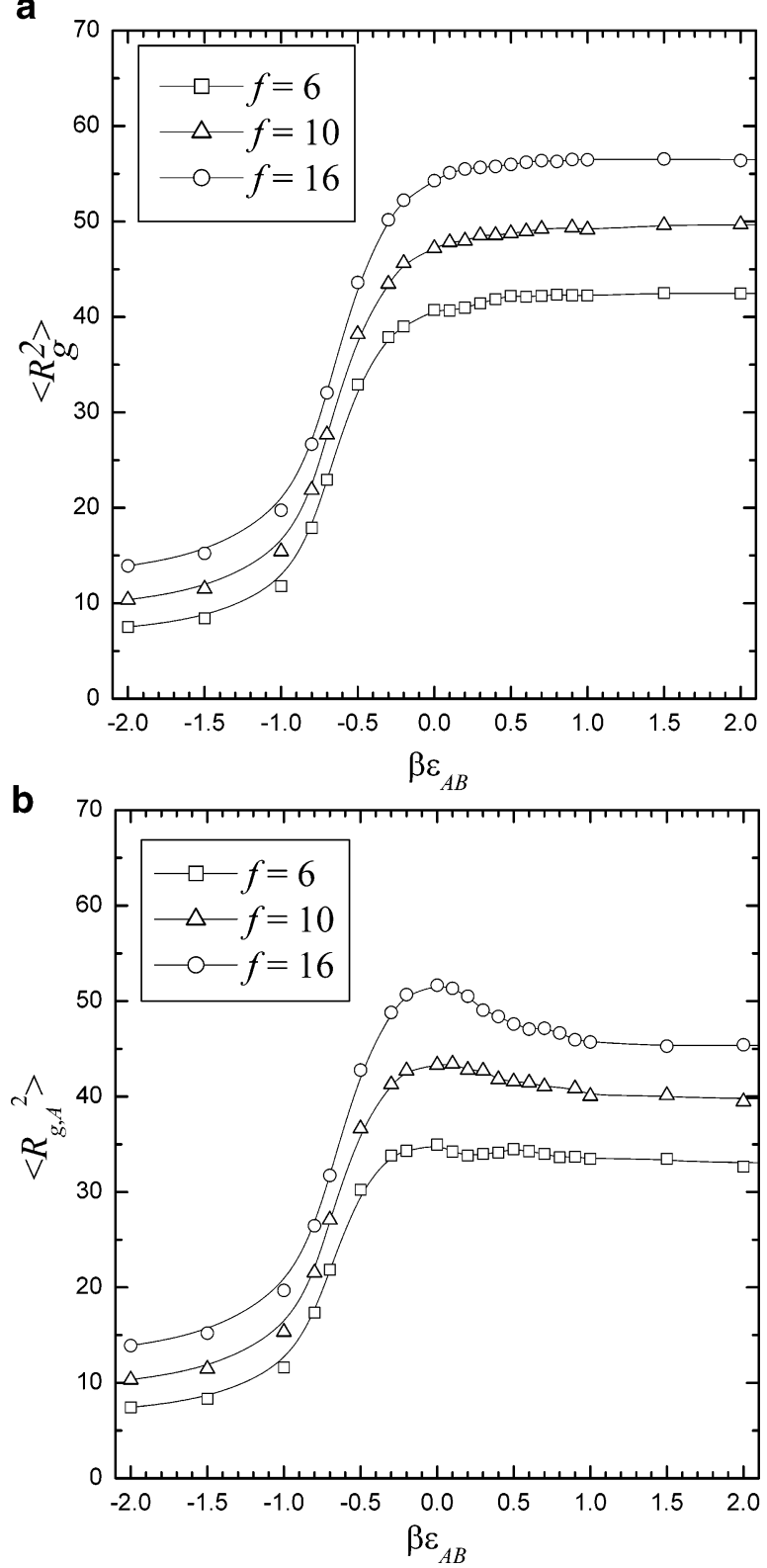

Figure 1. Variation of radius of gyration with the $A-B$ interaction for various arm numbers at a fixed arm length $(L$ = 20): (a) for the whole star copolymer and (b) for the $A$ block arms. All the lengths are scaled by the diameter of the bead $\sigma$.

$=-0.2$ and $\beta \epsilon_{A B}=-0.48$ also leads to the result of $v=$ 0 . Different theta states may correspond to different molecular conformations. If one analyzes the monomer pair correlation function such as $g_{A A}, g_{B B}$, and $g_{A B}$, different outcomes may emerge for different theta states. Nevertheless, $v=0$ is a reasonable measure of solvent quality.

As $\beta \epsilon_{A B}<-0.611$, the star copolymer is in a poor solvent condition, and it has a collapsed conformation. This inference is consistent with the variation of the radius of gyration with $\beta \epsilon_{A B}$. As shown in Figure 1a, the maximum slope of $\left\langle R_{\mathrm{g}}{ }^{2}\right\rangle$ occurs at $\beta \epsilon_{\mathrm{AB}}=-0.6$. The radius of gyration decreases rapidly toward a constant as the attraction between $A$ and $B$ blocks $\left(\epsilon_{A B}<0\right)$ is increased. When the interaction is repulsive, $\epsilon_{A B} \geq 0$, however, the variation of the radius of gyration with $\epsilon_{A B}$ is insignificant for a given arm number. It indicates that the excluded-volume interaction plays a more important role than that of the short-range $A-B$ seg- 
ment repulsion in determining $\left\langle R_{\mathrm{g}}{ }^{2}\right\rangle$. If there exists no segregation within a star copolymer, the radius of gyration associated with $A$ (or $B$ ) block, $\left\langle R_{\mathrm{g}, A^{2}}{ }^{2}\right.$, is expected to demonstrate behavior similar to that of $\left\langle R_{\mathrm{g}}{ }^{2}\right\rangle$. With decreasing $A-B$ segment attraction $\left(\epsilon_{A B}<\right.$ $0)$, Figure 1b shows that $\left\langle R_{\mathrm{g}, A}{ }^{2}\right\rangle$ grows rapidly and reaches a maximum at $\epsilon_{A B}=0$ (a homoarm star polymer). As the repulsion is increased further $\epsilon_{A B}>0$, however, a gradual decline of $\left\langle R_{\mathrm{g}, A^{2}}\right\rangle$ is observed. The extent increases with arm number. This consequence implies that repulsion between $A$ and $B$ blocks leads to a change in the conformation of $A$ (or $B$ ) block.

The relative position of the center of mass associated with $A$ (or $B$ ) block from the star core $\left(\mathbf{x}_{A}\right)$ is an indication of the conformational change of $A$ block. When $A-B$ block segregation takes place, the center of mass associated with $A$ block is no longer in the vicinity of the center of the star copolymer (star core). It shifts away from the star core, and hence $\left\langle R_{\mathrm{g}, A}{ }^{2}\right\rangle$ is lowered. To verify the above statement, the variation of the mean-square center of mass of $A$ block $\left\langle\mathbf{x}_{A}{ }^{2}\right\rangle$ (relative to star core) with $\beta \epsilon_{A B}$ is illustrated in Figure $2 \mathrm{a}$ for a given arm length $L=20$. Note that $\left\langle\mathbf{x}_{A}{ }^{2}\right\rangle$ consists of contributions due to arm fluctuation, excluded-volume expansion, and segregation. Since the arm fluctuation is getting significant with decreasing arm number, $\left\langle\mathbf{x}_{A}{ }^{2}\right\rangle$ is generally higher for smaller $f$.

For a given arm number, the conformations of the stars in $A-B$ segment attraction and repulsion regimes are quite different. For larger arm number, $f=16$, the segment density is rather high, and the fluctuation of $\mathbf{x}_{A}$ is therefore strongly hindered. In the attractive regime, an approximately symmetrical sphere (with respect to the star core) is formed. When the attraction is weakened, the excluded-volume interactions expand the polymer and lead to a rise in $\left\langle\mathbf{x}_{A}{ }^{2}\right\rangle$. The $A-B$ segment repulsions induce the Janus segregation and thus increase $\left\langle\mathbf{x}_{A}{ }^{2}\right\rangle$ furthermore. For smaller arm number, $f=6,\left\langle\mathbf{x}_{A}{ }^{2}\right\rangle$ also grows in the repulsive regime due to excluded-volume expansions and intramolecular segregation. However, in the attractive regime, there exists a competition between $A-B$ segment attraction-induced collapse and asymmetric segment distribution around the star core. At dilute segment density, the collapse toward to star core is no longer a better way to increase $A-B$ segment contact because the volume available in the vicinity of the star core is smaller than that of the outer shell. As a consequence, the star core may be pushed outside instead of being located at the center of the star copolymer. An asymmetric globule (with respect to the star core) is thus formed. The collapse gives a small value of $\left\langle\mathbf{x}_{A}{ }^{2}\right\rangle$ while the asymmetry results in a rise in $\left\langle\mathbf{x}_{A}{ }^{2}\right\rangle$. A minimum is therefore observed slightly lower than the $\Theta$-point at lower arm number. Figure 2a clearly shows that $\left\langle\mathbf{x}_{A}{ }^{2}\right\rangle$ always grows as $A-B$ repulsion is increased. Nonetheless, $\left\langle\mathbf{x}_{A}{ }^{2}\right\rangle$ of $f=16$ grows faster than those of $f=6$ and 10. This consequence reveals the increasing tendency of intramolecular segregation for a star copolymer with higher arm number. Figure $2 \mathrm{~b}$ depicts the variation of $\left\langle\mathbf{x}_{A}{ }^{2}\right\rangle$ with the arm number $f$ at very strong $A-B$ repulsion $\left(\beta \epsilon_{A B}=100\right)$. For $\beta \epsilon_{A B} \gg 1$, the segregation effect reaches its maximum. An increase in arm number leads to a decrease in the arm fluctuation effect but an increase in the segregation effect. As a result, $\left\langle\mathbf{x}_{A}{ }^{2}\right\rangle$ declines rapidly first, reaches a minimum, and then rises toward a plateau. The minimum takes place at $f=10$, owing to
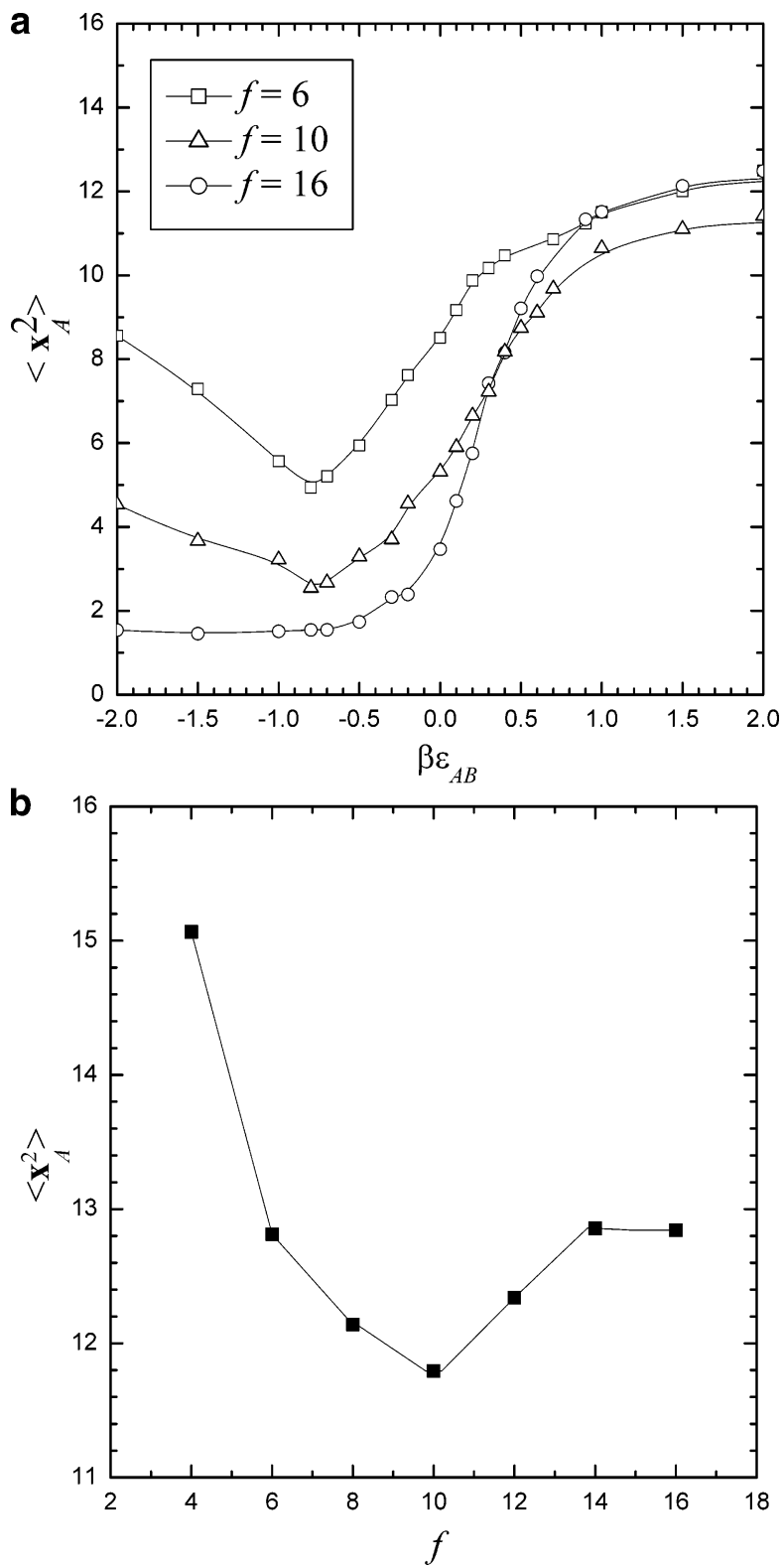

Figure 2. Mean-square distance from the center of mass associated with $A$ block arms to the star core is plotted against (a) the $A-B$ interaction for various arm numbers and against (b) the arm number for $\beta \epsilon_{A B}=100$.

the competition between arm fluctuation and intramolecular segregation.

As we know, the coordination number for hexagonal closest packing of hard spheres is 12 (when $\varsigma=1 \mathrm{in} \mathrm{eq}$ 1). However, in our work $\varsigma=1.4$; the largest accessible value of $f$, identified as $f_{\max }$, can reach 18 by random testing. It is plausible to conclude that the true $f_{\max }$ should be a little over 18 . Since $f_{\max } \approx 18$, there is still space for the first bead in an arm to move around for $f$ $=16$. In general, the relaxation time of the inner part of the star is expected to be much longer than that of the outer parts. However, it is found that the autocorrelation functions of the first and the second bond vectors originated from the core for $f=16$ are about $40000 \mathrm{MC}$ steps/bead and $20000 \mathrm{MCs} / \mathrm{bead}$, respectively. Our simulation length $\left(\geq 10^{8} \mathrm{MCs} / \mathrm{bead}\right)$ are well over the calculated relaxation times. Therefore, we believe that the outer parts as well as the inner parts of the stars are equilibrated in our simulation conditions. 
B. Degree of Segregation. The conformational properties $\left\langle R_{\mathrm{g}, A}{ }^{2}\right\rangle$ and $\left\langle\mathbf{x}_{A}{ }^{2}\right\rangle$ display qualitatively some characteristics associated with intermolecular segregation of a heteroarm star copolymer. To investigate the extent of segregation quantitatively, one must define the degree of segregation within a heteroarm star copolymer. As a first approach, the degree of segregation can be characterized by the mean-square separation between $A$ and $B$ blocks

$$
S_{1}=\frac{\left\langle\left(\mathbf{x}_{A}-\mathbf{x}_{B}\right)^{2}\right\rangle}{2\left\langle\mathbf{x}_{A}{ }^{2}\right\rangle}
$$

To remove the direct influences associated with molecular architecture, such as arm length, the mean-square separation $\left\langle\left(\mathbf{x}_{A}-\mathbf{x}_{B}\right)^{2}\right\rangle$ is scaled by the mean-square distance from $A$ (or $B$ ) block center to star copolymer core. The latter varies with segment-segment interaction $\epsilon_{A B}$, arm number $f$, and arm length $L$.

Since $S_{1}=1-\left\langle\mathbf{x}_{A} \cdot \mathbf{x}_{B}\right\rangle /\left\langle\mathbf{x}_{A}{ }^{2}\right\rangle$, one can also define another degree of segregation as

$$
S_{2}=\frac{\left\langle\mathbf{x}_{A} \cdot \mathbf{x}_{B}\right\rangle}{\left\langle\mathbf{x}_{A}{ }^{2}\right\rangle}
$$

Evidently, $-1 \leq S_{2} \leq 1$ and thus $0 \leq S_{1} \leq 2$. For a phantom polymer, the center locations of $\mathbf{x}_{A}$ and $\mathbf{x}_{B}$ are independent of each other, and thereby their correlation is zero, $\left\langle\mathbf{x}_{A} \cdot \mathbf{x}_{B}\right\rangle=0$. As a consequence, $S_{1}=1$ and $S_{2}=$ 0 . Following eq 8 , a similar degree of segregation can be defined as

$$
S=\left\langle\frac{\mathbf{x}_{A}}{\left|\mathbf{x}_{A}\right|} \cdot \frac{\mathbf{x}_{B}}{\left|\mathbf{x}_{B}\right|}\right\rangle=\langle\cos \theta\rangle
$$

Note that $-1 \leq S \leq 1$ and $S=0$ for a phantom heteroarm star copolymer. The degree of segregation may vary with strength of $A-B$ interaction $\epsilon_{A B}$, arm number $f$, and arm length $L$. If $A$ and $B$ blocks are attracted toward each other, the angle between $\mathbf{x}_{A}$ and $\mathbf{x}_{B}(\theta)$ is less than $\pi / 2$, and thus one has $S>0$. On the contrary, the repulsion yields the separation between $A$ and $B$ blocks. Since $\theta>\pi / 2$, the intramolecular segregation results in $S<0$.

The degree of segregation is adopted to examine the separation between $A$ and $B$ block arms within a heteroarm star copolymer. The variation of the degree of segregation $S_{2}$ (or $S_{1}$ ) with $\beta \epsilon_{A B}$ is depicted in Figure 3 . In the regime of strong $A-B$ segments attraction, Figure 3a shows that there exists strong positive correlation between $\mathbf{x}_{A}$ and $\mathbf{x}_{B}$. Since $S_{1}=1-S_{2}$, this result also means the mean separation between $A$ and $B$ blocks is small compared to the mean separation from the block center to the star core, owing to collapsed conformations. In addition, the aforementioned facts imply that $A$ and $B$ segments are uniformly mixed, but the star core is pushed away from the centers of both blocks. With increasing $\beta \epsilon_{A B}$, the correlation declines and the separation ratio grows. At $\beta \epsilon_{\mathrm{AB}} \approx-0.5, S_{1} \simeq 1$ and $S_{2} \simeq 0$ reveal that the excluded-volume interaction approximately cancels out the $A-B$ segment attraction. Note that $\mathrm{d} S_{1} / \mathrm{d} \beta \epsilon_{A B}\left(-\mathrm{d} S_{2} / \mathrm{d} \beta \epsilon_{A B}\right)$ also displays a maximum near $\beta \epsilon_{\mathrm{AB}} \simeq-0.6$, which is close to the $\Theta$-solvent condition based on the integral measure of solvent quality, eq 5 . These points are expected to coincide for infinitely long arm length. For $\beta \epsilon_{\mathrm{AB}} \gtrsim-0.5$, the fact that
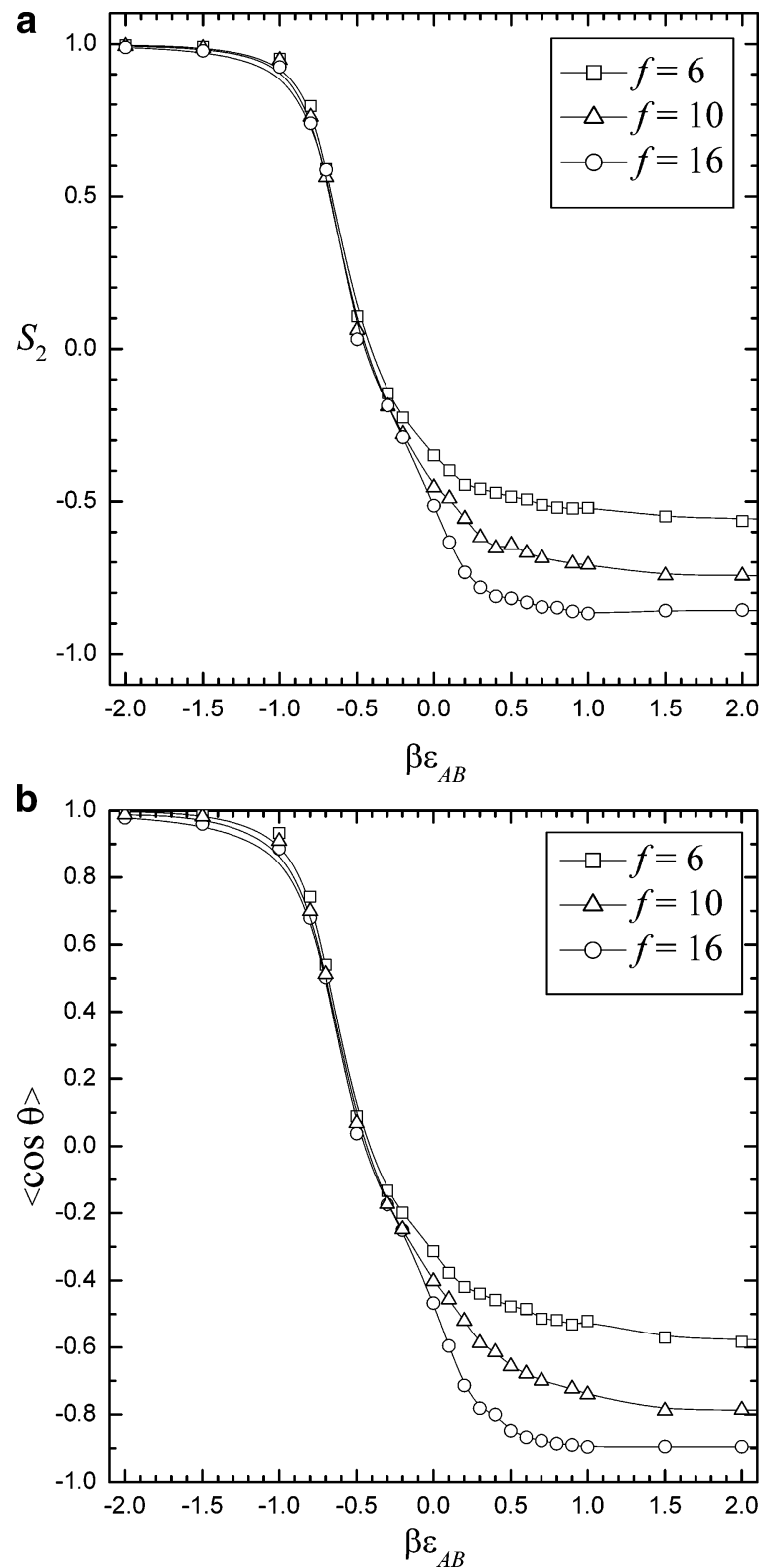

Figure 3. Variation of the degree of segregation (a) $\left\langle\mathbf{x}_{A} \cdot \mathbf{x}_{B}\right\rangle /$ $\left\langle\mathbf{x}_{A}{ }^{2}\right\rangle$ and (b) $\langle\cos \theta\rangle$ with the $A-B$ interaction for various arm numbers at a fixed arm length $(L=20)$.

$\mathbf{x}_{A}$ and $\mathbf{x}_{B}$ correlation becomes negative indicates the occurrence of intramolecular segregation is due to excluded-volume interactions and chain connectedness. The $A-B$ segment repulsion strengthens the segregation furthermore. Because of its short-ranged nature, the degree of segregation reaches an asymptotic value that must be greater than -1 (perfectly aligned in opposite direction). Another definition of degree of segregation $S(\langle\cos \theta\rangle)$ displays very similar behavior, as shown in Figure 3b.

The effect of arm number $f$ on the degree of segregation is demonstrated in Figure $3 a, b$ for various arm numbers at a given arm length $L=20$. When $A-B$ segments are well mixed due to strong $A-B$ attraction, the influence of arm number is insignificant. However, as the attraction is weak enough, the excluded-volume interaction becomes dominant and the difference in arm number takes effect. Figure 3a,b clearly shows that as the arm number is increased, the degree of segregation rises. Increasing arm number means raising segment density within a star polymer because the number of 


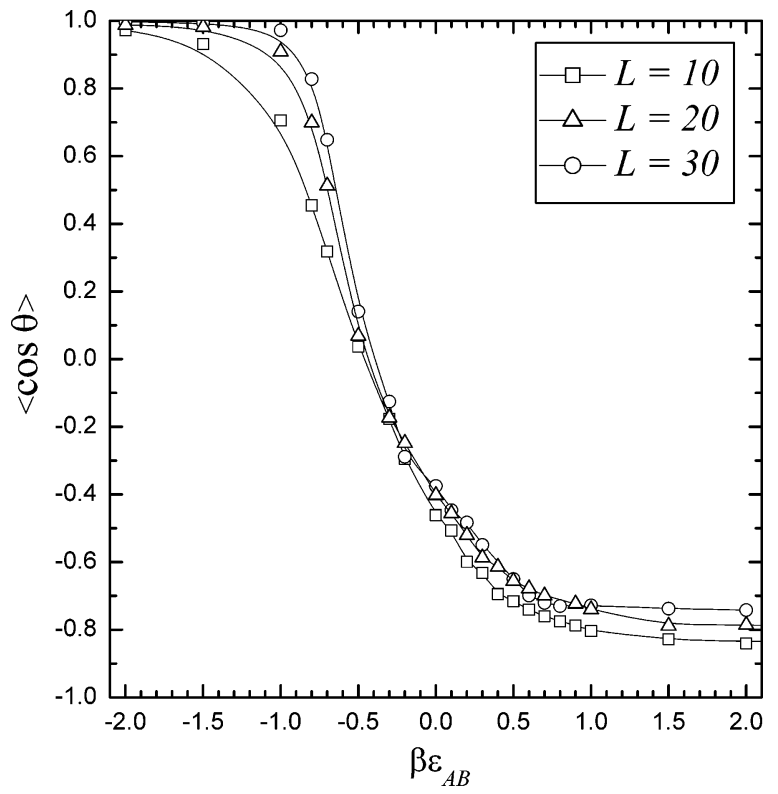

Figure 4. Variation of the degree of segregation $\langle\cos \theta\rangle$ with the $A-B$ interaction for various arm lengths at a fixed arm number $(f=10)$.

segment grows more rapidly than the radius of gyration does. In a mixture of $A$ - and $B$-type monomers, the short-ranged repulsion is able to result in phase separation. In a star copolymer, different extents of segregation may occur instead due to the competition between $A-B$ segment repulsion and conformational entropy. Here we define the extent of intramolecular Janus segregation as the degree of segregation exceeding that associated with a homoarm star copolymer $\left(\epsilon_{A B}=0\right)$. For $f=6$, the free volume available to an arm is large enough. The conformational entropy contribution is more important than that of $A-B$ repulsion, and therefore the Janus segregation is weak. For $f=16$, on the contrary, the repulsion dominates over the entropy, and the Janus segregation is more evident. In other words, the extent of Janus segregation rises with increasing arm number. In contrast, the influence of arm length on the Janus segregation is insignificant. Figure 4 demonstrates the variation of degree of segregation $\left(S_{2}\right)$ with $\beta \epsilon_{A B}$ for various arm lengths at a given $\operatorname{arm}$ number $f=10$. In general, the degree of segregation grows slightly with increasing arm length despite of the fact that the segment density actually declines with increasing arm length. This consequence indicates that the shortranged $A-B$ segment repulsions offer their major influences on intramolecular Janus segregation in the vicinity of the star core. The chain connectedness yield a relatively weak segregation effect. The snapshot of the morphology of a heteroarm star copolymer at different $A-B$ interactions due to $A-B$ block incompatibility is shown in Figure 5. The intramolecular Janus segregation is evidently displayed at $\beta \epsilon_{A B}=0.4$ and 0.8 while it is not clear at $\beta \epsilon_{A B}=0$.

C. Selective Solvent. In a selective solvent, the collapse of $B$ block arms can lead to the intramolecular segregation. Two possible molecular morphologies may be formed: Janus-like or micelle-like conformations. The latter consists of a hydrophobic core made by a $B$ segment globule and a hydrophilic shell made by arms of $A$ segment. Previous researchers often implied the formation of micelle-like morphology for intramolecular segregation within a heteroarm star copolymer. The

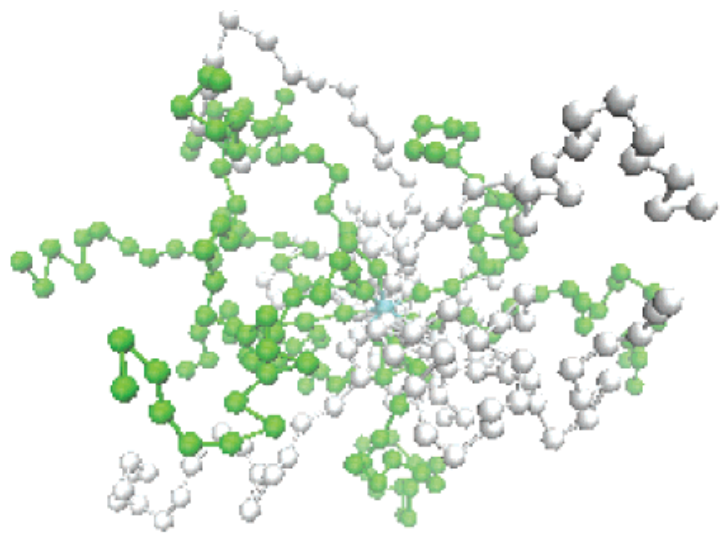

(a)

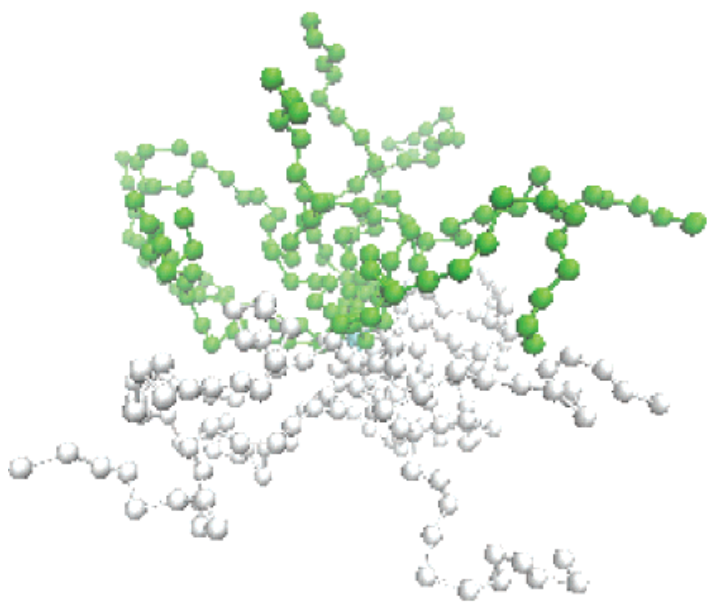

(b)

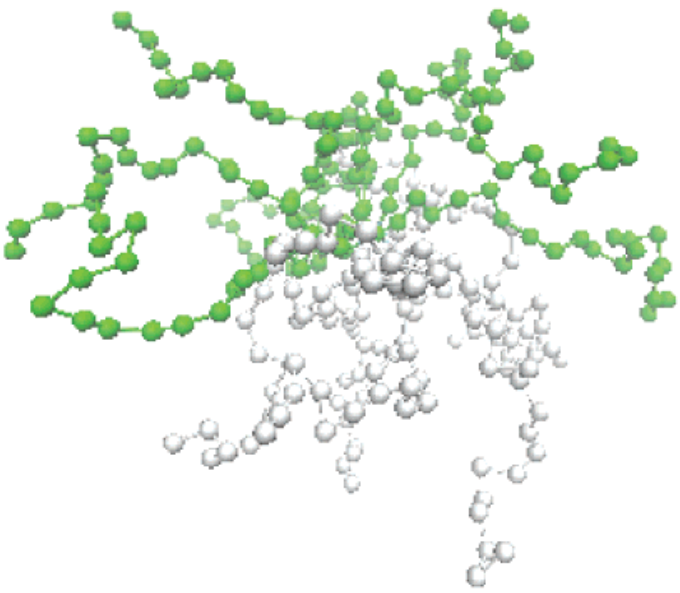

(c)

Figure 5. Snapshots of a heteroarm star copolymer with $f=$ 16 at different $A-B$ interactions are shown to display the intramolecular Janus segregation due to block incompatibility. (a) $\beta \epsilon_{A B}=0.0$, (b) $\beta \epsilon_{A B}=0.4$, and (c) $\beta \epsilon_{A B}=0.8$.

degree of segregation defined in eq 8 or 9 is a good indicator for determining the morphology. The degree of segregation approaches -1 for the Janus-like segregation while it is close to zero for the micelle-like segregation. Our simulation results confirm that heteroarm star copolymers adopt the Janus segregation.

For a selective solvent with $\epsilon_{A A}=\epsilon_{A B}=0$ and $\epsilon_{B B}<$ 0 , the partial bare second virial coefficients are $b_{A A}=$ $b_{A B}=1$. As $\beta \epsilon_{B B}<-0.35$, one has $b_{B B}<0$. That is, the $B$ block arms are in $\Theta$-solvent condition at $\beta \epsilon_{\mathrm{BB}} \simeq-0.35$. The theta value estimated here is in good agreement 


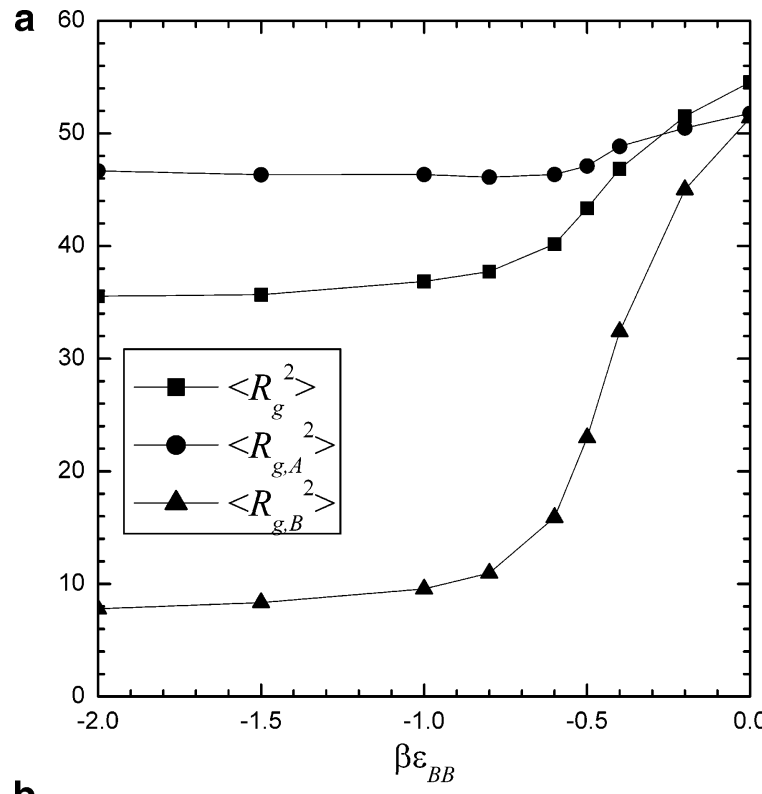

b

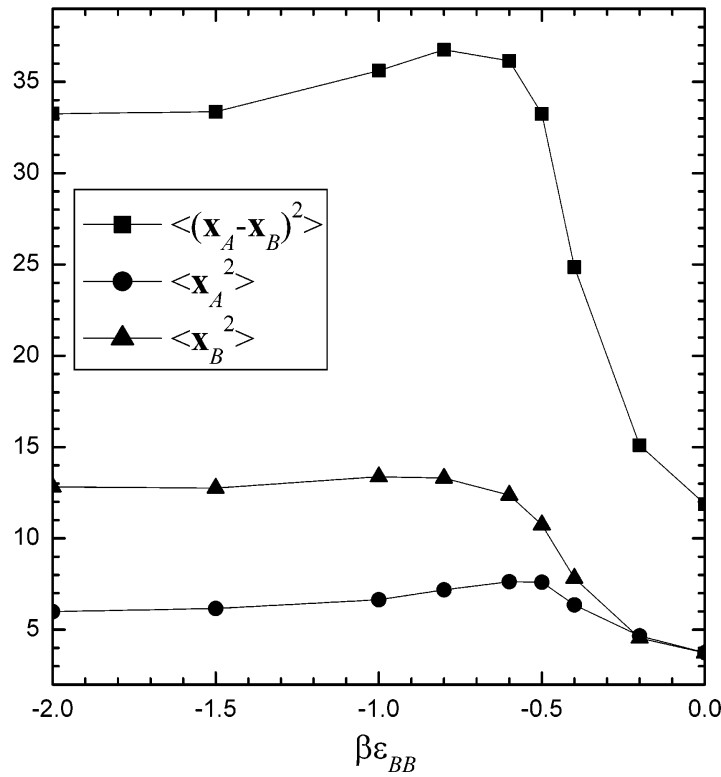

Figure 6. (a) Variation of the radius of gyrations of the whole star copolymer $\left\langle R_{\mathrm{g}}{ }^{2}\right\rangle, A$ block arms $\left\langle R_{\mathrm{g}, A}{ }^{2}\right\rangle$, and $B$ block arms $\left\langle R_{\mathrm{g}, B}{ }^{2}\right\rangle$ with the $B-B$ interaction. (b) Variation of the meansquare distance from the block center to the star core, $\left\langle\mathbf{x}_{A}{ }^{2}\right\rangle$ and $\left\langle\mathbf{x}_{B}{ }^{2}\right\rangle$, and the center-to-center separation between $A$ and $B$ blocks, $\left\langle\left(\mathbf{x}_{A}-\mathbf{x}_{B}\right)^{2}\right\rangle$, with the $B$ segment attraction for $f=$ 16 and $L=20$.

with other estimates. ${ }^{22}$ Figure 6 a shows that the maximum slopes of $\left\langle R_{\mathrm{g}, B}{ }^{2}\right\rangle,\left\langle R_{\mathrm{g}, A}{ }^{2}\right\rangle$, and $\left\langle R_{\mathrm{g}}{ }^{2}\right\rangle$ occur at $\beta \epsilon_{\mathrm{BB}}$ $\simeq-0.4$. Note that the radius of gyration of $A$ block arms with $\beta \epsilon_{B B}<0$ is slightly smaller than that with $\beta \epsilon_{B B}=$ 0 , owing to the decrease of excluded-volume repulsions provided by $B$ block arms. Figure $6 \mathrm{~b}$ demonstrates further the mean-square distance from the block center to the star core $\left(\left\langle\mathbf{x}_{A}{ }^{2}\right\rangle\right.$ and $\left.\left\langle\mathbf{x}_{B}{ }^{2}\right\rangle\right)$ and the center-to-center separation between $A$ and $B$ blocks $\left(\left\langle\left(\mathbf{x}_{A}-\mathbf{x}_{B}\right)^{2}\right\rangle\right)$. Those characteristic distances grow first and then decline with decreasing $\beta \epsilon_{B B}$. One can evidently observe that there exist maxima in $\left\langle\mathbf{x}_{A}^{2}\right\rangle$ and $\left\langle\left(\mathbf{x}_{A}-\mathbf{x}_{B}\right)^{2}\right\rangle$. This result reflects the competition among influences associated with collapse of $B$ block arms, intramolecular segregation, and arm fluctuations. The growth regime signifies the collapse of $B$ block arms and intramolecular segregation. In comparison with homoarm star polymers, the

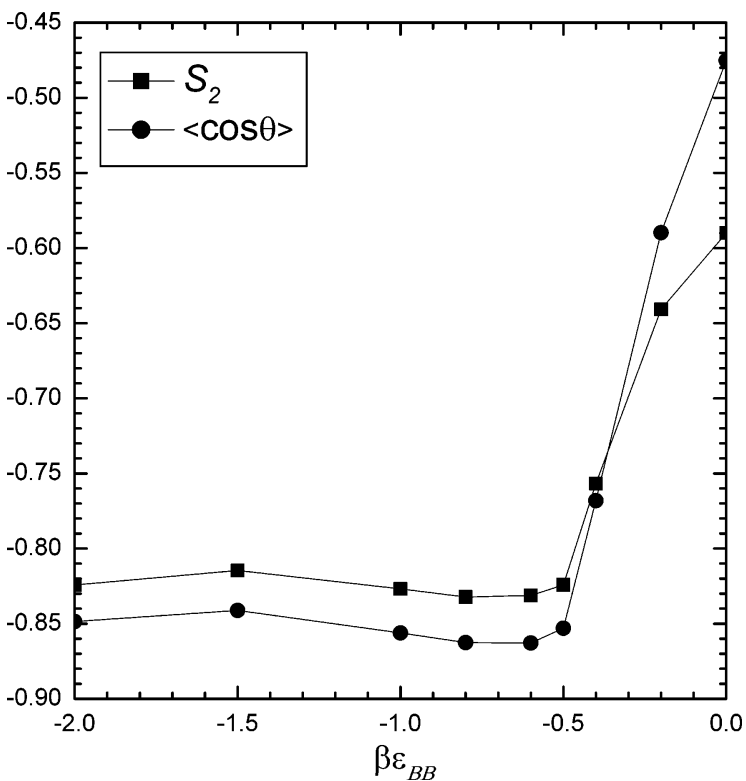

Figure 7. Degree of segregation in a selective solvent is plotted against the $B$ segment attraction for $f=16$ and $L=$ 20 .

Janus segregation reduces the space available for arm fluctuations of $A$ or $B$ block arms. It also pushes the mean block center away from the star core. The mean separation between $A$ and $B$ blocks is therefore increased. However, once the arms of $B$ block have been in a poor solvent condition, the space volume occupied by $B$ blocks shrinks significantly. Consequently, arm fluctuations associated with $A$ block rise slightly and result in a weak decrease of $\left\langle\mathbf{x}_{A}{ }^{2}\right\rangle$ and $\left\langle\left(\mathbf{x}_{A}-\mathbf{x}_{B}\right)^{2}\right\rangle$. The maximum values of the negative derivative, such as $\left.-\mathrm{d}\left\langle\mathbf{x}_{i}^{2}\right\rangle / \mathrm{d} \beta \epsilon_{B B}\right)$, correspond to the $\Theta$-solvent condition associated with $B$ blocks. That is, the attraction among $B$ segments is balanced by their excluded-volume repulsion. At even stronger $B$ segment attractions, the maximum values of the positive derivatives are located at about $\beta \epsilon_{B B}=-1$. In accord with the integral measure of solvent quality, $v<0$ only when $\beta \epsilon_{B B}<-0.99$. Thereby, it can be regarded as the conformation crossover due to the balance between the $B$ block attraction and the $A$ block repulsion.

Figure 7 shows the variation of the degree of segregation with the $B$ segment attraction for $f=16$ and $L=$ 20 . Both definitions display that the Janus segregation grows with increasing the $B$ segment attraction. However, it seems that a maximum extent is reached and then $-S$ (or $-S_{2}$ ) declines slightly. Again, this result can be attributed to the reason that $A$ block arms invade the space yielded by the $B$ block globule. Figure 8 illustrates the snapshots of the polymer morphology. The increase in the $B$ segment attraction $\beta \epsilon_{B B}$ from $-0.2,-0.6$, to -1.0 clearly demonstrates the gradual formation of the $B$ block globule. The angle $(\theta)$ spans from $A$ block center to $B$ block center is toward $\pi$ and yields the Janus morphology. The consequence of the Janus segregation is responsible for the formation of the multimolecular micelles by heteroarm star copolymers and can be explained by the effective intermolecular potential.

D. Effective Interaction Potential and Second Virial Coefficient. The physical significance of the intramolecular Janus segregation can be manifested through the effective interaction potential $U_{\text {eff }}(\xi)$ and 

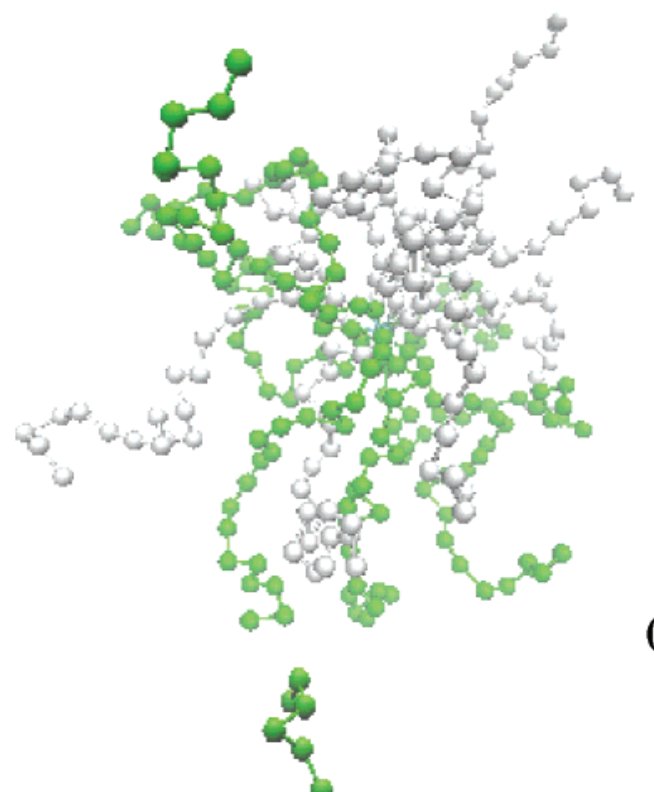

(a)

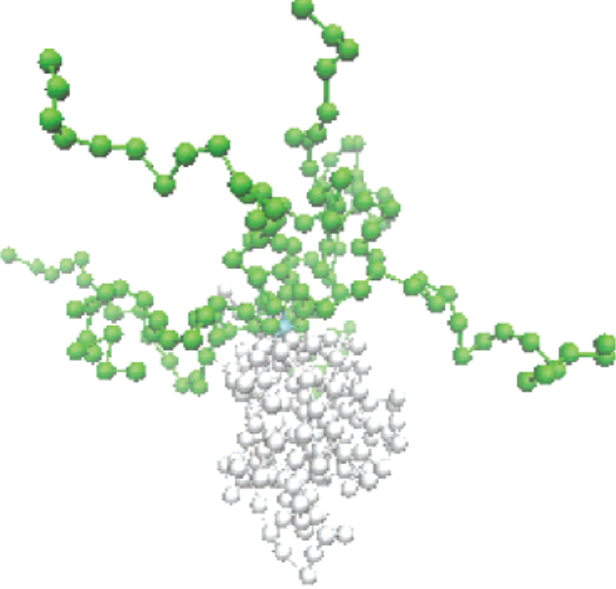

(b)

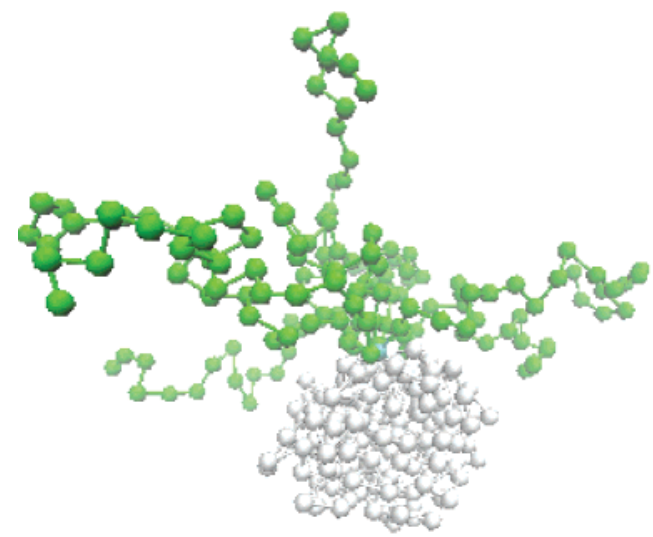

(c)

Figure 8. Snapshots of a heteroarm star copolymer with $f=$ 16 at different $B$ segment attraction are shown to display the intramolecular Janus segregation due to selective solvents. (a) $\beta \epsilon_{B B}=-0.2$, (b) $\beta \epsilon_{B B}=-0.6$, and (c) $\beta \epsilon_{B B}=-1.0$. The bead with white color represents $B$-type monomer.

the second virial coefficient $B_{2}$ between two star copolymers. In selective solvents for $B$-type monomers, we consider the effect of solvent quality $\beta \epsilon_{B B}$ on both $\beta U_{\text {eff }}$ and $B_{2}^{*}=B_{2} /\left(4 \pi R_{\mathrm{g}}{ }^{3} / 3\right)$. Owing to the excluded-volume interactions between segments, the effective interaction between two homoarm star copolymers $\left(\epsilon_{B B}=0\right)$ is always repulsive. As shown in Figure 9, the potential of mean force is monotonically increased with decreasing intermolecular distance $\xi$. As the $B$ segment attraction

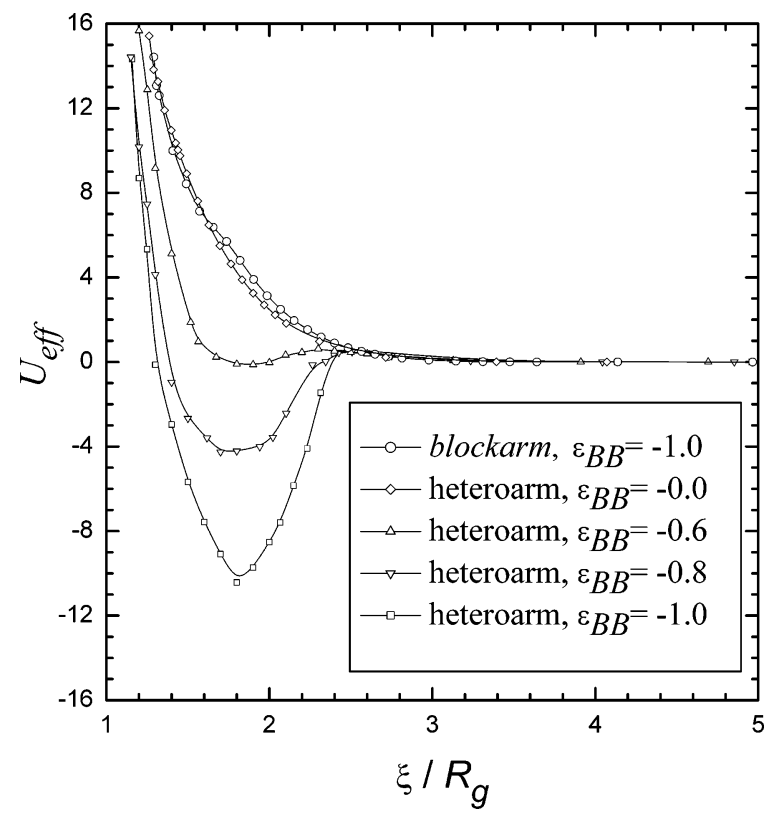

Figure 9. Potential of mean force $\left(U_{\text {eff }}\right)$ between two heteroarm star copolymers is plotted against the intermolecular distance $\xi / R_{\mathrm{g}}$ for various $B$ segment interactions. For comparison, $U_{\text {eff }}$ associated with a blockarm star copolymer at $\beta \epsilon_{B B}=$ -1.0 is also given.

Table 1. Scaled Second Virial Coefficient as a Function of the $B$ Segment Attraction

\begin{tabular}{rccccc}
\hline$\beta \epsilon_{B B}$ & $B_{2}^{*}$ & $\beta \epsilon_{B B}$ & $B_{2}^{*}$ & $\beta \epsilon_{B B}$ & \multicolumn{1}{c}{$B_{2}^{*}$} \\
\hline 0.0 & 7.86 & -0.4 & 7.52 & -0.8 & -141 \\
-0.1 & 7.63 & -0.5 & 6.39 & -0.9 & -4790 \\
-0.2 & 7.73 & -0.6 & 5.14 & -1.0 & -33800 \\
-0.3 & 7.60 & -0.7 & -24.9 & &
\end{tabular}

is increased, the repulsion between the two polymers declines. When the attraction between $B$ segments is strong enough, i.e., $\beta \epsilon_{B B} \simeq-0.6$, a minimum appears at $\xi \approx 1.8 R_{\mathrm{g}}$ in $U_{\text {eff }}(\xi)$, indicating the existence of intermolecular attraction at some separations, just like the Lennard-Jones potential. This consequence reveals that two star polymers with Janus segregation are in favor of arranging themselves with the two $B$ block globules in close neighborhood configuration. The well depth of the effective interaction potential increases rapidly with increasing $B$ segment attraction. The net interaction between two heteroarm star copolymers is determined by the competition between globule-globule attractions and excluded-volume repulsions. It can be manifested through the second virial coefficient. As illustrated in Table 1 , for $\beta \epsilon_{B B}>-0.5, B_{2}$ is about $6-8$ times of the characteristic polymer volume $4 \pi R_{\mathrm{g}}{ }^{3} / 3$, which varies with $\beta \epsilon_{B B}$ as well. However, the fact that $B_{2}^{*}$ drops rapidly from positive to negative values at $-\beta \epsilon_{B B}=0.6-0.7$ indicates that strong intermolecular attraction occurs due to intramolecular Janus segregation. Note that for $B$ type homoarm star polymers a value of $\beta \epsilon_{\mathrm{BB}} \approx 0.3$ is expected for a vanishing virial coefficient. For a $A B$ heteroarm star copolymer, as an attractive interaction between $B B$ implicitly induces a repulsive interaction between $A B$, thus a more negative parameter is necessary as compared to the case. The Janus segregation and micellization in selective solvents have the same thermodynamic origin. The formation of Janus structures in individual stars may facilitate the association process and speed up the micellization kinetics. Consequently, like the micelles formed by a few 


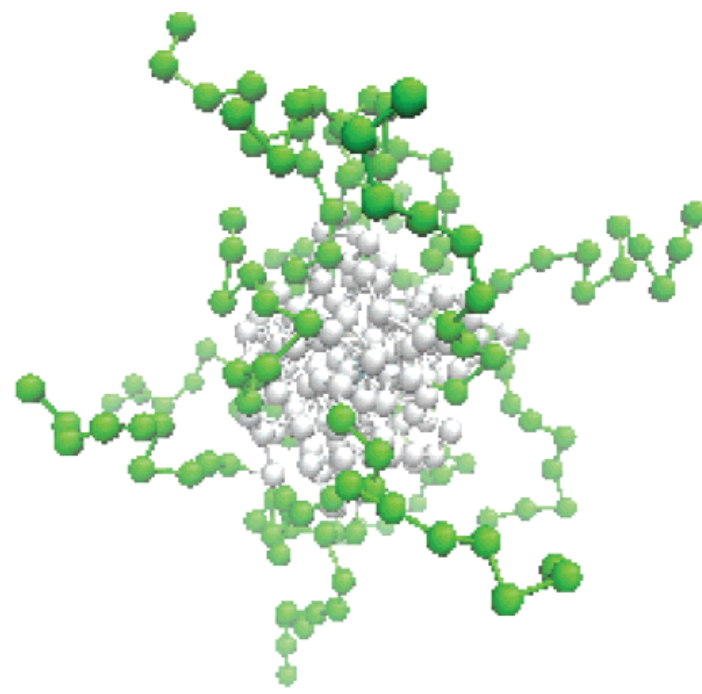

(a)

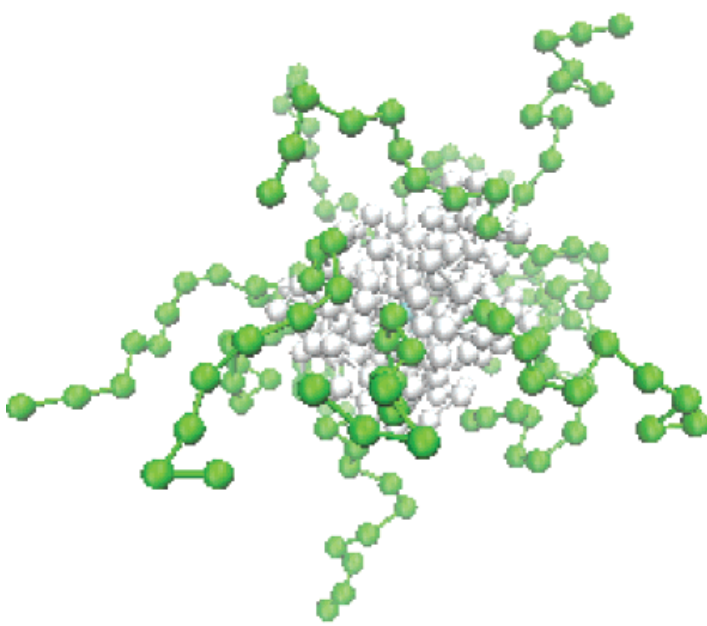

(b)

Figure 10. Two representative snapshots of a blockarm star copolymer with $f=16$ at $\beta \epsilon_{B B}=-1.0$ are shown to display the micelle-like segregation. The bead with green color represents $A$-type monomer.

surfactants or diblock linear copolymers, Janus-like heteroarm star copolymers can self-assemble to form multimolecular micelles.

It should be mentioned that our simulation of the second virial coefficient may slightly underestimate the association tendency of stars in selective solvents. Theoretically the method employed here should provide correct value of $B_{2}$ as long as all possible conformations for the star polymers have been sampled. It is possible that some energy-driven conformations associated with a pair of Janus stars may not be sampled in our simulation scheme. In that case, an even stronger attractive interaction, i.e., more negative $B_{2}$, should be obtained.

In this study, our MC simulations clearly show that the intramolecular segregation can take place within a heteroarm star copolymer under appropriate conditions of block incompatibility or selective solvents. The degree of segregation grows with increasing arm numbers. The segregation morphology adopts the Janus-like conformation, which faciliates the formation of multimolecular micelles. Recently, however, the unimolecular micelle morphology (collapsed core-extended shell) for a single heteroarm star copolymer is proposed based on the AFM observation of $\mathrm{PS}_{7}-\mathrm{PVP}_{7}$ adsorbed on mica. ${ }^{12}$ This inference is based on the assumption that the adsorption process does not alter the polymer conformation. Nonetheless, the Janus-like structure in bulk solvent may be easily transformed into the unimolecular micelle morphology upon adsorption if the collapsed core is maintained. In contrast, the unimolecular micelle morphology can be formed by the blockarm star copolymer. Figure 10 shows two representative snapshots of the blockarm star copolymer with a collapsed core by $B$ segment and extended hairy arms by $A$ segment at $\beta \epsilon_{B B}$ $=-1.0$. Just like homoarm star polymers, our calculations show that the effective interaction potential between two blockarm star copolymers is always repulsive, as illustrated in Figure 9, even at $\beta \epsilon_{B B}=-1.0$. This consequence indicates that it is rather difficult for star copolymers possessing a unimolecular micelle morphology to form multimolecular micelles, owing to the excluded-volume interactions provided by the extended shell of hairy arms.

Acknowledgment. This research is supported by National Council of Science of Taiwan. Computing time, provided by the National Center for High-Performance Computing of Taiwan, is gratefully acknowledged.

\section{References and Notes}

(1) Meier, M.; Gohy, J.; Fustin, C.; Schubert, U. J. Am. Chem. Soc. 2004, 126, 11517.

(2) Haag, R. Angew. Chem., Int. Ed. 2004, 43, 278.

(3) Liu, M.; Kono, K.; Frechet, J. J. Controlled Release 2000, 65, 121

(4) Anastasiadis, S.; Retsos, H.; Pispas, S.; Hadjichristidis, N.; Neophytides, S. Macromolecules 2003, 36, 1994.

(5) Heise, A.; Hedrick, J.; Frank, C.; Miller, R. J. Am. Chem. Soc. 1999, $121,1$.

(6) Pispas, S.; Hadjichristidis, N.; Potemkin, I.; Khokhlov, A. Macromolecules 2000, 33, 1741.

(7) Ishizu, K.; Uchida, S. Prog. Polym. Sci. 1999, 24, 1439.

(8) Hadjichristidis, N.; Pitsikalis, M.; Pispas, S.; Iatrou, H. Chem. Rev. 2001, 101, 3747.

(9) Pitsikalis, M.; Pispas, S.; Mays, J.; Hadjichristidis, N. Adv Polym. Sci. 1998, 135, 1.

(10) Voulgaris, D.; Tsitsilianis, C.; Esselink, F. J.; Hadziiloannou, G. Polymer 1998, 39, 6429 .

(11) Kiriy, A.; Gorodyska, G.; Minko, S.; Stamm, M.; Tsitsilianis, C. Macromolecules 2003, 36, 8704.

(12) Gorodyska, G.; Kiriy, A.; Minko, S.; Tsitsilianis, C.; Stamm, M. Nano Lett. 2003, 3, 365.

(13) Zifferer, G. J. Chem. Phys. 1999, 110, 4668.

(14) Di Cecca, A.; Freire, J. Macromolecules 2002, 35, 2851.

(15) Molina, L.; Freire, J. Macromolecules 1999, 32, 499.

(16) Vlahos, C. H.; Horta, A.; Hadjichristidis, N.; Freire, J. J. Macromolecules 1995, 28, 1500.

(17) Havránková, J.; Limpouchová, Z.; Procházka, K. Macromol. Theory Simul. 2003, 12, 512.

(18) Sheng, Y.-J.; Jiang, S.; Tsao, H.-K. Macromolecules 2002, 35 , 7865 .

(19) Sheng, Y.-J.; Chen, J. Z.-Y.; Tsao, H.-K. Macromolecules 2002, $35,9624$.

(20) Sheng, Y.-J.; Hsu, P.-H.; Chen, J. Z.-Y.; Tsao, H.-K. Macromolecules 2004, 37, 9257.

(21) Vasilevskaya, V. V.; Khalatur, P. G.; Khokhlov, A. R. Macromolecules 2003, 36, 10103.

(22) Dautenhahn, J.; Hall, C. K. Macromolecules 1994, 27, 5399.

(23) Allen, M. P.; Tildesley, D. J. Computer Simulations of Liquids; Oxford University Press: New York, 1987.

(24) Harismiadis, V. I.; Szleifer, I. Mol. Phys. 1994, 81, 851.

(25) McQuarrie, D. A. Statistical Mechanics; Harper \& Row: New York, 1976

(26) Yamakawa, H. Modern Theory of Polymer Solutions; Harper and Row: New York, 1971.

(27) Olaj, O. F.; Neubauer, B.; Zifferer, G. Macromolecules 1998, 31,4342

MA050596G 